\title{
Conocimiento Especializado de un Profesor de Álgebra Lineal y Espacios de Trabajo Matemático
}

\author{
Mathematics Teacher's Specialised Knowledge and the Mathematical \\ Working Spaces of a Linear Algebra's Teacher
}

\author{
Diana Vasco Mora* \\ Nuria Climent ${ }^{* *}$ \\ Dinazar Escudero-Ávila ${ }^{* * *}$ \\ Miguel A. Montes ${ }^{* * * *}$ \\ Miguel Ribeiro ${ }^{* * * * *}$
}

\begin{abstract}
Resumen
En este trabajo indagamos sobre las posibles relaciones entre los resultados del análisis de una sesión de clase con dos modelos teóricos: el modelo Mathematics Teacher's Specialised Knowledge (MTSK) y los Espacios de Trabajo Matemático (ETM). De este modo, discutimos el conocimiento especializado (MTSK) que evidencia en una sesión sobre la multiplicación de matrices un profesor universitario de álgebra lineal y el espacio de trabajo matemático (ETM) idóneo del profesor en esta sesión. El conocimiento evidenciado por el profesor parece explicar las génesis que el profesor prima en el trabajo matemático que propone. Así, el análisis con ambos modelos permite comprender mejor la actividad matemática que propicia el profesor y cómo se explica desde su conocimiento.
\end{abstract}

Palabras clave: Espacios de Trabajo Matemático. Conocimiento Especializado del Profesor de Matemáticas. Álgebra Lineal. Matrices. Estudio de Caso.

\section{Abstract}

\footnotetext{
* Doctora por la Universidad de Huelva (Didáctica de la Matemática), Universidad de Huelva (UHU), Huelva, España. Profesora Titular Agregada de Universidad Técnica Estatal de Quevedo (UTEQ), Quevedo, Los Ríos, Ecuador. Dirección postal: Calle Quinta, entre C y D, 608, Ciudadela San José Sur, Quevedo, Los Ríos, Ecuador. E-mail: dianav350@yahoo.com.

** Licenciada en Matemáticas, Universidad de Sevilla (US), y Doctora en Psicopedagogía, Universidad de Huelva (UHU). Profesora Titular de Universidad de Didáctica de la Matemática, Universidad de Huelva (UHU), Huelva, España. Dirección postal: Facultad de Ciencias de la Educación, Campus El Carmen, Avenida 3 de Marzo, s/n, 21071. Huelva, España. E-mail: climent@uhu.es.

*** Maestra en Ciencias; CINVESTAV; Doctora por la Universidad de Huelva (Didáctica de la Matemática), Universidad de Huelva (UHU), Huelva, España. Dirección postal: Facultad de Ciencias de la Educación, Campus El Carmen, Avenida 3 de Marzo, s/n, 21071. Huelva, España. E-mail: eadinazar@ hotmail.com.

Licenciado en Matemáticas por la Universidad de Sevilla (US), y Doctor por la Universidad de Huelva (Didáctica de las Matemáticas), Universidad de Huelva (UHU), Huelva, España. Profesor de Didáctica de la Matemática, Universidad de Huelva (UHU), Huelva, España. Dirección postal: Facultad de Ciencias de la Educación, Campus El Carmen, Avenida 3 de Marzo, s/n, 21071. Huelva, España. E-mail: miguel.montes@ddcc.uhu.es.

Licenciado en Matemáticas, Universidade da Beira Interior (Portugal) y Doctor por la Universidad de Huelva (Didáctica de las Matemáticas), Universidad de Huelva (UHU), Huelva, España. Bolseiro Posdoutorado Cnpq. E-mail: cmribas78@gmail.com.
} 
In this study, we approach the possible relationships between the analysis results of a classroom session with two different theoretical models: the Mathematics Teacher's Specialized Knowledge (MTSK), and the Mathematical Working Spaces (MWS). We also discuss the specialized knowledge that an university teacher of Linear Algebra deploys in a session about matrix product, and the ideal MWS of the teacher in this session. The knowledge showed explains the different genesis that the teacher prioritizes in the mathematical work he proposes. Thereby, the analysis with both models allows us to understand in depth the mathematical activity that the teacher fosters and how it is sustained by his knowledge.

Keywords: Mathematical Working Spaces. Mathematics Teacher's Specialised Knowledge. Linear Algebra. Matrices. Case Study.

\section{Introducción}

El conocimiento matemático desde la perspectiva de su enseñanza está íntimamente relacionado con las facultades que posee el profesor para realizar representaciones que ayuden a desarrollar habilidades matemáticas en los estudiantes (CHARALAMBOUS, 2009). Esta relación entre las habilidades del profesor y el desarrollo que pueden tener sus estudiantes ha generado un interés por comprender el tipo particular de conocimiento que necesita el profesor de matemáticas, así como por la forma en que se adquiere y desarrolla dicho conocimiento (SÁNCHEZ, 2011). En general, los modelos de conocimiento del profesor de matemáticas pretenden comprender el funcionamiento y la estructura del conocimiento que tiene el profesor de matemáticas y que utiliza para la gestión de su práctica en el aula.

A efectos de discutir aspectos del conocimiento especializado que se observa en la práctica de un profesor universitario cuando enseña el contenido de multiplicación de matrices hemos empleado el modelo analítico denominado conocimiento especializado del profesor de matemáticas (Mathematics Teacher's Specialised Knowledge - MTSK) (CARRILlO et al., 2013). Por su parte, el Espacio de Trabajo Matemático (ETM) se constituye en un ambiente pensado y organizado para facilitar el trabajo matemático (KUZNIAK; RICHARD, 2014), donde el profesor utiliza estrategias y promueve tareas que articulan los componentes del ETM.

La discusión de la acción del profesor en el aula nos permite analizar el conocimiento especializado que se moviliza, además de aportar información sobre los espacios de trabajo matemático que el profesor propicia (ETM idóneo). En este artículo relacionamos el ETM idóneo con el conocimiento del profesor, en el caso de un profesor universitario de álgebra lineal en una sesión de clase sobre multiplicación de matrices. Este proceso de consideración conjunta de ambos constructos permitirá evidenciar aquellos elementos que ambos modelos 
realzan en el análisis, alcanzando, tras éste, una comprensión más profunda del conocimiento que sustenta la actividad matemática que el profesor propone generar en el aula.

\section{Marco teórico}

Para contribuir a una mejora de la formación y de las prácticas, un foco en el conocimiento que pone en juego el profesor en el aula, y que se manifiesta en los espacios de trabajo que propone durante las sesiones de clases, se torna central. Para el estudio de dicho conocimiento adoptamos la perspectiva del MTSK (CARRILLO et al., 2013), que considera la especialización del conocimiento del profesor respecto de la enseñanza de la matemática. Complementariamente, para el análisis de la actividad matemática que propone el profesor hemos seguido la teoría del ETM, que intenta describir el trabajo matemático desarrollado por los estudiantes en la escuela. A continuación abordamos con más detalle cada uno de estos marcos, deteniéndonos en aquellos elementos teóricos clave para nuestro análisis de la sesión.

\subsection{Mathematics Teacher's Specialised Knowledge (MTSK)}

El MTSK es un modelo analítico de tipo descriptivo que permite elaborar una interpretación integral del conocimiento especializado del profesor (ROJAS; FLORES; CARRILLO, 2015), tomando en cuenta las diferencias que existen en cuanto a los criterios de validez y las diferentes facetas del conocimiento del profesor (conocimiento matemático y didáctico específico).

El MTSK (Figura 1) considera, así, dos dominios del conocimiento del profesor: el dominio del Conocimiento Matemático (MK), entendido como un conocimiento amplio y profundo de las matemáticas escolares, y el dominio del Conocimiento Didáctico del Contenido (PCK), referido al conocimiento sobre el contenido matemático como objeto de enseñanza y aprendizaje. Hay que destacar que el énfasis en el PCK se refiere a los conocimientos en donde el contenido matemático condicione la enseñanza y el aprendizaje de las matemáticas, excluyendo así los conocimientos pedagógicos generales en contextos de actividades matemáticas (ESCUDERO-ÁVILA et al., 2015).

En cada uno de los dominios anteriores se diferencian tres subdominios (ver Figura 1). La visión del MTSK como un modelo sobre el conocimiento especializado del profesor se complementa, de forma explícita, con las concepciones del profesor sobre la matemática y su enseñanza y aprendizaje. 


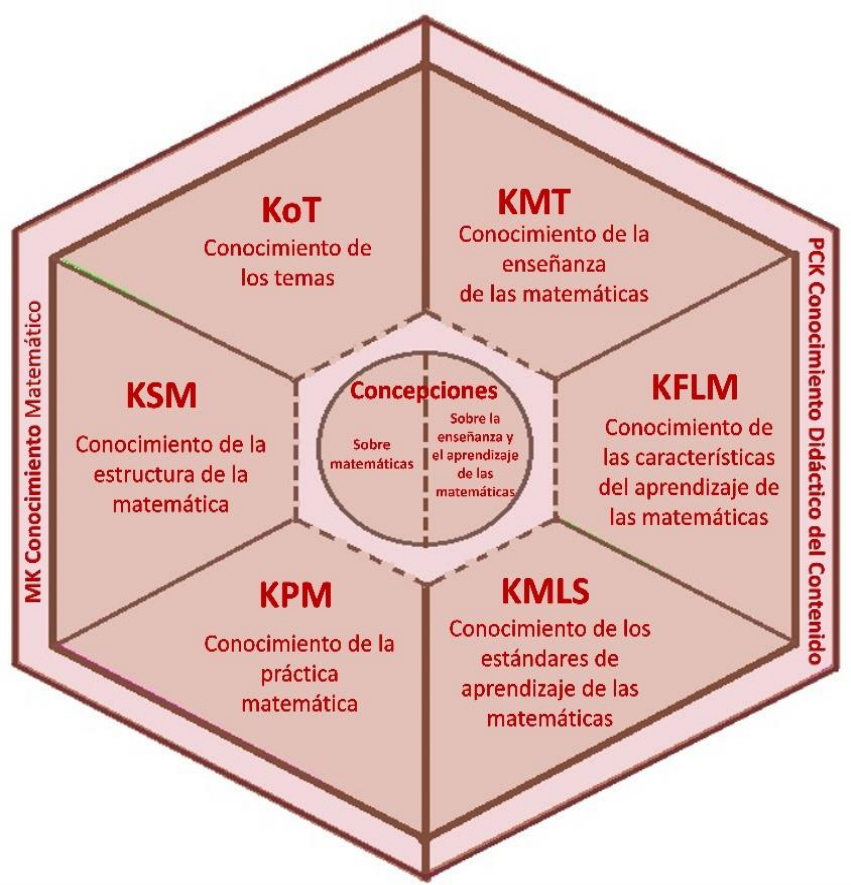

Figura 1 - Dominios y subdominios del MTSK (CARRILLO et al., 2013)

A continuación describimos cada uno de los seis subdominios, poniendo énfasis en el KoT, KFLM y KMT, puesto que son los que discutiremos más profundamente en el apartado del análisis ${ }^{1}$.

En el conocimiento de los temas (KoT) se ubica el conocimiento del profesor acerca de usos y aplicaciones de un tema matemático y el conocimiento sobre modelos atribuibles a un tema, es decir, el conocimiento sobre la fenomenología y aplicaciones asociadas a un determinado contenido matemático. Se considera también el conocimiento sobre propiedades (de un objeto matemático) y sus fundamentos, donde se incluye el conocimiento sobre las bases y la exhaustividad del empleo de una propiedad, ligado al tema a estudiar. Además, forman parte del KoT, el conocimiento para describir o caracterizar un concepto, incluyendo los ejemplos e imágenes asociados, es decir, el conocimiento sobre la(s) definición(es) de un objeto matemático, así como el conocimiento acerca de los distintos registros de representación o formas en que se puede representar un tema, al igual que el conocimiento de la notación y el lenguaje matemático. En este subdominio se incluyen, también, conocimientos sobre procedimientos matemáticos estándar y alternativos asociados a un determinado contenido matemático.

El conocimiento de la estructura de la matemática (KSM) engloba los conocimientos del profesor sobre las relaciones entre distintos contenidos matemáticos (MONTES et al.,

\footnotetext{
${ }^{1}$ Una descripción más extensa del MTSK se puede encontrar en (FLORES-MEDRANO et al., 2016) - en este mismo volumen.
} 
2013), en particular las conexiones inter-conceptuales (MARTíNEZ et al., 2011) e implica ver el contenido en perspectiva, las matemáticas elementales desde un punto de vista avanzado (con respecto al contenido que se atiende) y las matemáticas avanzadas desde un punto de vista elemental.

El conocimiento de la práctica matemática (KPM), por su parte, incluye conocimiento de las formas de crear o producir que son características del trabajo matemático (CARRILLO et al., 2013).

Incluido en el Conocimiento Didáctico del Contenido, el conocimiento de la enseñanza de las matemáticas (KMT) se refiere al conocimiento del profesor sobre teorías personales o institucionalizadas de enseñanza, recursos materiales y virtuales, así como su conocimiento sobre actividades, tareas y ejemplos para enseñar un contenido matemático.

En el conocimiento de las características del aprendizaje de las matemáticas (KFLM) se incluye el conocimiento sobre las formas de aprendizaje inherentes a un contenido matemático en particular o a la matemática en general, y de las características de aprendizaje derivadas de la interacción de los estudiantes con el contenido matemático. Se enfoca el contenido matemático como objeto de aprendizaje e incluye el conocimiento sobre formas de aprendizaje (asociado a teorías personales del profesor o institucionalizadas), fortalezas y dificultades asociadas al aprendizaje de un contenido y las motivaciones de los estudiantes respecto del mismo.

Finalmente, el conocimiento de los estándares de aprendizaje de las matemáticas (KMLS) incluye el conocimiento de las especificaciones del plan de estudios, la progresión de un año a otro, así como objetivos y medidas de desempeño desarrolladas por entidades externas tales como asociaciones profesionales e investigadores (CARRILLO et al., 2013).

\subsection{Espacios de Trabajo Matemático (ETM)}

El ETM (Figura 2) surgió como Espacio de Trabajo Geométrico catalogado como un ambiente organizado para la resolución de problemas geométricos (KUZNIAK, 2011); posteriormente fue ampliado a otros espacios de la matemática denominándose Espacio de Trabajo Matemático al ambiente pensado y organizado para permitir el trabajo de los individuos que resuelven problemas matemáticos (KUZNIAK; RICHARD, 2014).

Este marco propone modelizar la actividad matemática (en principio de un sujeto) atendiendo a dos planos/niveles: el epistemológico (en relación estrecha con los contenidos matemáticos del ámbito estudiado), y el cognitivo (que concierne al pensamiento del sujeto 
que resuelve tareas matemáticas). Estos niveles, epistemológico y cognitivo, estructuran los ETM y ayudan a comprender la circulación de los conocimientos entre los componentes del plano (KUZNIAK; RICHARD, 2014).

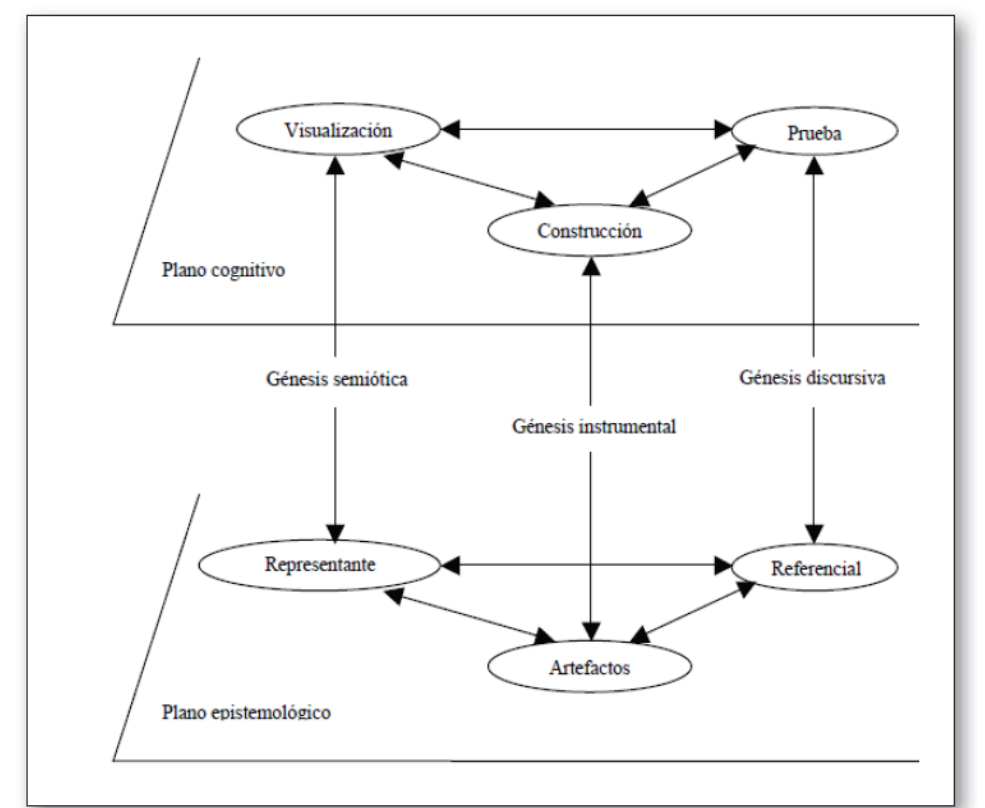

Figura 2 - Espacio de Trabajo Matemático (ETM) y sus génesis (KUZNIAK, 2011)

En el plano epistemológico se diferencian tres componentes: representante (o representamen), artefacto y referencial. La idea de representamen, fundamentada en representaciones semióticas (DUVAL, 1995) se refiere a la noción de signo, que pueden ser íconos, índices, símbolos o gráficas, y se relaciona con el objeto matemático bajo formas más o menos abstractas (HENRÍQUEZ; MONTOYA, 2015); la idea de artefacto, empleado como un medio para la acción, se refiere a herramientas, pudiendo ser material o un sistema simbólico; y el sistema teórico o referencial se refiere a conocimiento de procedimientos, propiedades, definiciones y fundamentos.

El plano cognitivo recoge la actividad del sujeto cognitivo y se conceptualiza a través de tres procesos que permiten dar un sentido a los signos y a los objetos: la visualización, relacionada con un proceso de estructuración de las informaciones aportadas por los diagramas y los signos (KUZNIAK; RICHARD, 2014), la construcción, relativa a una significación mediada por instrumentos y la prueba, entendida como un proceso discursivo que permite construir argumentaciones.

La imbricación de ambos planos se produce a través de transformaciones denominadas génesis (semiótica, instrumental y discursiva), que permiten pasar de los niveles potenciales de un objeto matemático (plano epistemológico) a su uso por un individuo (plano cognitivo). La génesis semiótica relaciona el signo con la visualización, la cual les confiere a dichos 
signos el estatus de objetos matemáticos (KUZNIAK; RICHARD, 2014); la génesis instrumental, relaciona el artefacto con el proceso de construcción (KUZNIAK; RICHARD, 2014; HENRÍQUEZ; MONTOYA, 2015) transformándose el artefacto en herramienta; y a través de la génesis discursiva se otorga un significado al referencial usándolo en el razonamiento matemático (KUZNIAK; NECHACHE, en prensa).

La articulación entre esas génesis se puede representar mediante tres planos verticales en la Figura 2. Así podemos diferenciar el plano Semiótico-Instrumental (plano de descubrimiento), el plano Instrumental-Discursivo (plano de razonamiento), y el plano Semiótico-Discursivo (plano de comunicación). En cada caso se enfatiza la activación de las dos génesis correspondientes y se relaciona con una fase del trabajo matemático implementado en la ejecución de una tarea: descubrimiento y exploración, justificación y razonamiento, presentación y comunicación (KUZNIAK; RICHARD, 2014). En el análisis del ETM interesa especialmente la articulación de las dimensiones y planos en torno a una actividad o tarea.

En el marco de los ETM se diferencia el ETM de referencia, ETM idóneo y ETM personal. El ETM de referencia (donde el hacedor de matemáticas es el sujeto epistemológico ideal) es aquel definido por una comunidad de individuos que se han puesto de acuerdo sobre un paradigma dado para formular problemas y organizar sus soluciones, privilegiando ciertas herramientas o formas de pensamiento. El ETM idóneo (referido al espacio de trabajo matemático implementado en una clase por el profesor) se organiza para permitir al alumno comprometerse permitiendo el trabajo en el paradigma correspondiente a la problemática considerada, no es fijo y debe modificarse continuamente ajustándose a restricciones locales, organizadas de manera válida. El ETM personal es aquel donde el profesor o el alumno dan un tratamiento matemático a un problema (KUZNIAK, 2011; KUZNIAK; RICHARD, 2014).

En este artículo nos ocupamos de las relaciones entre el ETM idóneo de un profesor en una sesión de clase y su conocimiento especializado.

\section{Marco metodológico}

Nuestra investigación se caracteriza por ser cualitativa e interpretativa y emplea un diseño de estudio de caso (YIN, 1989).

El estudio se realiza con un profesor universitario, al que llamaremos Jordy (J), que imparte un módulo de Álgebra Lineal en una carrera de Ingeniería en Ecuador. Jordy posee experiencia en la enseñanza de asignaturas de matemáticas, tanto en secundaria (17 años) 
como en el nivel universitario (9 años) e imparte Álgebra Lineal desde hace 4 años. El programa de estudios de la asignatura está previamente establecido y su punto de partida es el tema de Matrices y Determinantes (tipos, operaciones, resolución de sistemas de ecuaciones lineales y aplicaciones, complementadas con el estudio de determinantes); posteriormente se imparten temáticas como vectores en $\mathbf{R}^{2}, \mathbf{R}^{3}$ y $\mathbf{R}^{n}$, espacios vectoriales y transformaciones lineales. La clase analizada corresponde a la segunda sesión del tema de Matrices y Determinantes, dedicada a la multiplicación de matrices.

La recopilación de datos incluyó observaciones de clase (grabaciones en vídeo) y una entrevista semiestructurada. Dichos datos forman parte de una investigación más amplia (VASCO; CLIMENT; FLORES-MEDRANO, 2014; VASCO et al., en prensa). La finalidad de esta investigación es comprender el conocimiento que sustenta la enseñanza de un profesor universitario de Álgebra lineal en relación con dicha enseñanza. En ese sentido, no se ha hecho una elección del caso atendiendo a características especiales. Se busca comprender la situación de la enseñanza del Álgebra en el nivel y contexto en que se sitúa este profesor, a través del estudio en profundidad de un profesor.

En el artículo que aquí presentamos, en el que se mantiene la perspectiva de comprender la práctica del profesor a través de su conocimiento (analizado con el modelo MTSK), exploramos la posibilidad de introducir el análisis de la actividad matemática que propone el profesor, usando el modelo ETM, para así poder refinar la posible relación entre la enseñanza del profesor y el conocimiento especializado que se evidencia en su práctica.

Para esta exploración sobre el posible uso de los dos modelos de análisis, seleccionamos información en torno a una sesión de clase sobre multiplicación de matrices. El análisis de los datos que aquí se presentan se compone de dos partes atendiendo a un análisis de contenido (BRYMAN, 2004) análisis del conocimiento del profesor buscando evidencias en sus acciones y declaraciones que aludieran al conocimiento que sustenta su acción bajo el modelo MTSK, y 2) análisis de la actividad matemática que promueve el profesor en la sesión de cara a identificar el ETM idóneo. Para organizar el análisis identificamos episodios (momentos diferenciados de la clase por la temática que se aborda) y las tareas que el profesor propone a los alumnos.

Por tanto, en este trabajo presentamos los resultados del análisis conjunto del conocimiento de Jordy y el espacio de trabajo matemático idóneo implementado en la sesión.

\section{Resultados: ETM idóneo y MTSK evidenciado}


El ETM idóneo se ocupa de estudiar la actividad matemática que el profesor propicia en el aula. El trabajo matemático que propone el profesor objeto de nuestro estudio (Jordy), percibido a través del análisis de la sesión, se compone de un primer episodio que corresponde a la exploración de las condiciones y algoritmo de multiplicación de matrices, y un segundo episodio, que corresponde al trabajo sobre el álgebra de matrices. Las tareas planteadas por Jordy, si bien tienen un marcado énfasis procedimental, parecen pretender desarrollar una comprensión del procedimiento más allá de lo meramente repetitivo, profundizando en cuándo es posible desarrollar dicho algoritmo.

Hemos identificado tareas planteadas por el profesor durante la sesión de clases mencionada, paso necesario para el análisis del ETM idóneo implementado por éste. Asimismo, para el análisis de dicho ETM intentaremos determinar qué planos pretende el profesor abordar, así como qué génesis se ponen en juego. Somos conscientes de que el ETM idóneo no ha de coincidir con el que efectivamente desarrollen los estudiantes (LAVAL, 2015).

A continuación se presentan los episodios de la clase con las tareas en cada uno de ellos, que se corresponden con actividades propuestas por el profesor para los alumnos (Tabla $1)$.

Tabla 1 - Episodios y tareas identificados en la sesión de clases de Jordy.

\begin{tabular}{|l|l|}
\hline \multicolumn{1}{|c|}{ Episodios } & \multicolumn{1}{c|}{ Tareas } \\
\hline 1 - Producto de matrices & t1: Identificar cuándo es posible multiplicar matrices dadas por sus dimensiones \\
& t2: Multiplicar matrices dadas \\
\hline $2-$ Álgebra de matrices & t3: Calcular la imagen de una matriz por una función polinómica \\
\hline
\end{tabular}

En el epígrafe siguiente se describen, primeramente, cada uno de los episodios, acompañado, posteriormente, por el análisis del ETM idóneo implementado por el profesor y el conocimiento especializado que lo sustenta.

\subsection{Episodio 1 - producto de matrices}

Jordy comienza su clase pidiendo a los alumnos que recuerden que en la clase anterior vieron que es necesario que dos matrices tengan las mismas dimensiones para que se puedan sumar, y enseguida escribe una matriz $\mathrm{A}(2 \times 3)$ en la pizarra para indicar que en el caso del producto de matrices también se necesita cumplir con una condición. Complementa su explicación escribiendo tres matrices más de diferentes dimensiones, y a cada una la identifica como B cambiando sus dimensiones $(3 \times 1,3 \times 2,3 \times 4)$, con la finalidad de dejar claro a los 
estudiantes qué se necesita para la multiplicación de matrices. Posteriormente, hace referencia a las dimensiones que debe tener el resultado del producto de dos matrices.

A continuación, el profesor propone la tarea 1:

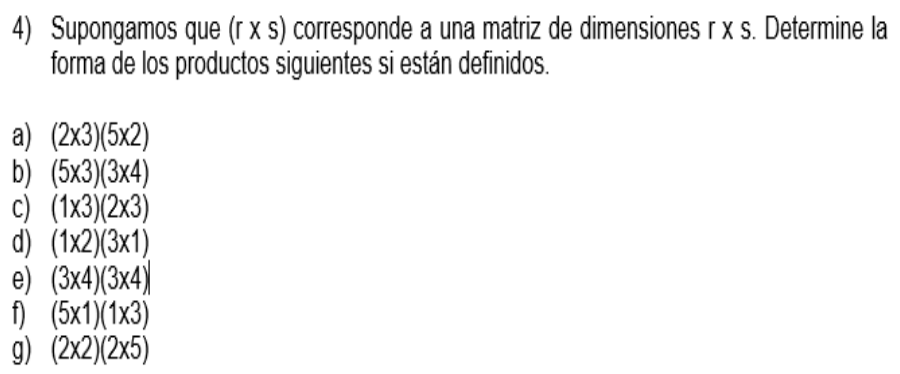

Figura 3 - Imagen de la tarea 1

El profesor elige algunos de los apartados de la tarea para discutirlos en gran grupo, preguntando a algunos alumnos, como aclaración de la misma.

Jordy: ¿Qué dice ahí? ¿Cuál es la orden?

Estudiante: Supongamos que rxs corresponde a una matriz de dimensiones $r \times s$. Determine la forma de los productos siguientes si están definidos.

J: $\quad$ A usted le plantean ahí por ejemplo $(3 \times 5)(4 \times 3)$ ¿está definido ese producto?

E: $\quad$ No.

J: ¿Por qué?

E: $\quad$ No coinciden las columnas y las filas.

J: $\quad$ No coincide el número de columnas de la primera matriz con el número de filas de la segunda, usted le escribe ahí no está definido el producto, no se puede realizar esa operación. Si le colocan este ejemplo de aquí $(2 \times 5)(5 \times 1)$ ¿Qué dice usted?

E: $\quad$ Sí.

J: $\quad$ Que sí se puede multiplicar, sí está definido y ¿cuáles son las dimensiones de la matriz resultante?

E: $\quad 2 \times 1$.

J: $\quad 2 \times 1$ ¿¿está claro?, resuelvan eso.

Durante la discusión, un estudiante comenta que "en la multiplicación de matrices sí cuenta el orden en el que estén ubicadas" y a partir de ello el profesor introduce la no conmutatividad del producto de matrices.

En la segunda parte del episodio (relativo a la tarea 2), el profesor explica a los alumnos cómo multiplicar dos matrices con ejemplos concretos y para ello emplea las matrices que ya tenía en la pizarra identificándolas como $A=\left[\begin{array}{ccc}2 & 3 & 1 \\ 4 & -5 & 0\end{array}\right], B=\left[\begin{array}{c}1 \\ -3 \\ 5\end{array}\right]$, $C=\left[\begin{array}{cc}2 & 3 \\ -1 & 5 \\ 7 & 0\end{array}\right] y \mathrm{D}=\left[\begin{array}{cccc}-1 & 0 & 3 & 4 \\ 5 & 2 & 3 & -1 \\ 2 & -2 & 5 & 0\end{array}\right]$ e indicando los pasos a desarrollar en el algoritmo para este caso. Algunos de los productos son realizados por los estudiantes en la pizarra. 
Finalmente, solicita a los estudiantes resolver el ejercicio 5 de la hoja de actividades propuestas que consiste en multiplicar matrices (Figura 4).

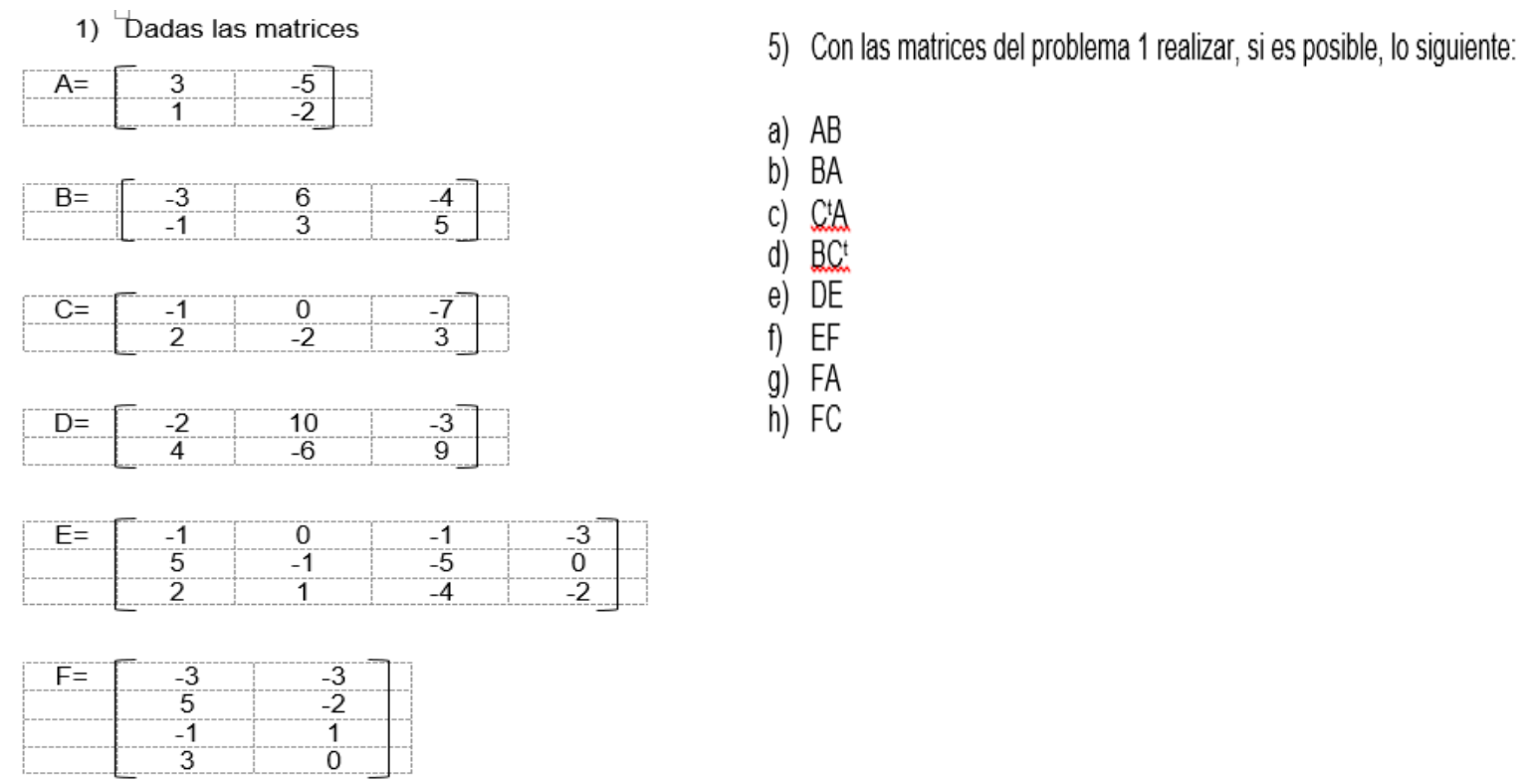

Figura 4 - Imagen de la tarea 2

Jordy enfatiza la consideración de las dimensiones de las matrices para poder realizar su producto. Dedica una parte de la sesión a tal fin, diseñando tareas específicas. Este énfasis parece apoyarse en su conocimiento de dificultades habituales de los alumnos. Así, el profesor sabe que los estudiantes cometen errores en la multiplicación de matrices por no considerar sus dimensiones, posiblemente inducidos por una falsa generalización del algoritmo de la suma de matrices al caso del producto (KFLM - fortalezas y dificultades asociadas al aprendizaje). La entrevista realizada a Jordy tras la sesión confirma este conocimiento:

J: $\quad$ El error que pueden cometer es que el chico piense que hay que multiplicar número por número, según la posición que está. Entonces, en la multiplicación de matrices, yo suelo insistir en las dimensiones, por eso al principio se les dice cómo determinar las dimensiones de una matriz y que sepan hacerlo bien para que no haya después confusión.

En relación con el plano epistemológico del ETM, hemos asociado la multiplicación de matrices como signo o representamen en lo relativo a su representación algebraica, como artefacto cuando es considerada como procedimiento y como referencial en lo relativo a sus propiedades (en este caso las condiciones sobre las dimensiones de las matrices implicadas, la caracterización de la dimensión de la matriz resultante y la no conmutatividad de dicho producto). Si bien somos conscientes de que el trabajo matemático que propone el profesor es básicamente procedimental, con énfasis en el algoritmo, destacamos algunos aspectos conceptuales en su tratamiento del procedimiento (las referidas propiedades) que enriquecerían el referencial teórico en el ETM. 
Interpretamos que la primera tarea propuesta (tarea 1) pretende contribuir a desarrollar la génesis discursiva, relativa a la justificación de cuándo es posible realizar la multiplicación de matrices. Hemos de considerar que en esta sesión se trata un contenido procedimental, por lo que creemos necesario precisar los elementos del ETM en relación con el trabajo sobre este tipo de contenido. Así, el razonamiento discursivo se referiría a la justificación del procedimiento y la discusión sobre su caracterización; mientras que asociamos a la génesis instrumental la aplicación del procedimiento para la obtención de un resultado, interpretando en este caso el procedimiento como herramienta simbólica.

Jordy presenta varios ejemplos de matrices en la tarea, con los que pone énfasis en dicha compatibilidad, y cuya elección se sustenta en su KMT (conocimiento, diversidad e importancia de ejemplos para la enseñanza), en términos de elegir aquellos ejemplos que activen la discusión sobre el tema en la línea pretendida. Los ejemplos seleccionados muestran un conocimiento que atiende a un criterio de variabilidad (FIGUEIREDO; CONTRERAS; BLANCO, 2009). Asimismo elige tres matrices de distintas dimensiones que pueden multiplicarse por la primera, y no considera ejemplos de matrices cuadradas, lo que puede asociarse, por un lado, a su cuidado en la elección de los ejemplos pero, por otro, ese cuidado imposibilitará que los estudiantes desarrollen un conocimiento asociado a la importancia de los casos particulares en y para la generalización. Estas elecciones están condicionadas por el conjunto de ejemplos que el profesor conoce, así como por su KoT acerca de matrices, especialmente en cuanto a los procedimientos aritméticos que las involucran.

En el transcurso de la tarea, y ante el comentario de un estudiante ("en la multiplicación de matrices sí cuenta el orden en el que estén ubicadas”), el profesor intenta que el alumno, que está en la génesis semiótica visualizando los ejemplos, pase a desarrollar un trabajo de tipo discursivo (en el sentido de enunciar el resultado de su observación en términos de una propiedad matemática). El profesor propicia el estudio general de la observación hecha por el estudiante, para dotarla de la entidad de propiedad de la multiplicación de matrices, ampliando el referencial teórico (la caracterización del producto de matrices) y promoviendo el razonamiento discursivo:

\section{E: $\quad$ O sea que en la multiplicación de matrices sí cuenta el orden}

J: $\quad$ ¿Cómo sería eso? ¿Cómo lo podrías decir? Allí hicieron $A \times B($ con $A(2 \times 3)$ y $B(3 \times 1))$, pero que si las colocamos al revés $B \times A((3 \times 1)(2 \times 3))$ no se puede multiplicar, en este caso ¿qué pasa?

E: $\quad$ No es conmutativa 
J: $\quad$ Correcto, en el producto de matrices no se cumple la propiedad conmutativa $(A \times B \neq$ $B \times A)$; no siempre es conmutativa, primero por las dimensiones y luego aunque se pudiese siendo matrices cuadradas, estas no siempre son conmutativas.

En este caso, el KoT del profesor le permite explorar esa propiedad como general, ampliando el caso evidente de que no siempre es posible realizar el producto en ambos sentidos. En una entrevista posterior a la sesión, muestra, además, conocer excepciones a la no conmutatividad:

J: $\quad$ Cuando es la matriz inversa, ahi es conmutativo, o sea, la matriz inversa por su matriz que siempre da la identidad. Y hay otros casos que a veces me han salido al azar.

Muestra de este modo cierta exhaustividad en el conocimiento de la propiedad, ya que no sólo la enuncia, sino que además, es cuidadoso en fundamentar dicho conocimiento y en la consideración de los casos - aunque no lo extienda a las particularidades de las situaciones que "le han salido al azar".

Pasamos a presentar el análisis de la tarea 2. Consideramos que, en este caso, ésta es la tarea clave de la sesión, ya que es aquella "en la que el docente focaliza la atención en el tema central de la clase" (HENRÍQUEZ; MONTOYA, 2015, p.59), siendo el objetivo de la misma el aprendizaje del algoritmo de multiplicación de matrices. Se pueden considerar las restantes tareas de la sesión como una tarea previa de familiarización con las propiedades (tarea 1), y una tarea posterior de aplicación de lo aprendido (tarea 3).

El trabajo matemático fomentado en esta sesión se centra en el dominio del algoritmo de multiplicación de matrices. Se pretende que los alumnos identifiquen en qué casos es posible realizar el producto y apliquen el algoritmo para calcular la matriz resultante. En este proceso se potencia la activación de un tipo de génesis instrumental, en el cual un procedimiento es usado como artefacto simbólico para obtener un resultado. El conocimiento que permite al profesor gestionar el trabajo matemático en esta tarea está sustentado en su KoT sobre el algoritmo, desde lo asociado a la dimensión puramente procedimental, hasta su conocimiento de las condiciones necesarias para multiplicar (compatibilidad de matrices). Vuelve a evidenciarse en la gestión de esta tarea en el aula el KFLM del profesor referido a errores de los alumnos en el algoritmo de multiplicación de matrices:

J: $\quad$ Antes de que haga la multiplicación revise por si acaso. [...] La ubicación de los factores es para que usted se equivoque menos. [...] Siempre es bueno definir las dimensiones de las matrices para evitar algún tipo de error en el producto.

Al igual que en el caso de la tarea anterior, la elección de las matrices que los alumnos multiplicarán, denota KMT (ejemplos de enseñanza), ya que tanto en las matrices elegidas, como en las combinaciones elegidas, podemos observar cómo fomenta la aparición de 
resultados ricos para su discusión, en términos de la compatibilidad de matrices, o de la no conmutatividad de las mismas (eligiendo tanto ejemplos como contraejemplos).

\subsection{Episodio 2 - álgebra de matrices}

Jordy indica a los alumnos que trabajarán el álgebra de matrices. Explica cómo a partir de una matriz $A=\left[\begin{array}{cc}2 & -1 \\ 5 & 1\end{array}\right]$ se pueden obtener $A^{2}=\left[\begin{array}{cc}-1 & -3 \\ 15 & -4\end{array}\right]$ y $A^{3}=\left[\begin{array}{cc}-17 & -2 \\ 10 & -19\end{array}\right]$, y procede a escribir una función $f(x)=2 x^{2}-5 x-3$, que le sirve como ejemplo para explicar cómo operar en el contexto de funciones con matrices como dominio. En el desarrollo de este ejemplo el profesor llama la atención sobre la naturaleza del término independiente -3:

J: $\quad$ En este -3 aquí colocamos la matriz identidad de orden $2 \times 2$ para poder sumar; si no, ese -3 no tiene forma de ser sumado acá, como estamos trabajando con matrices [...] al lado del 3 escribir la matriz identidad $2 \times 2$. Ahí tienen lo que ya vieron en la clase anterior: suma de matrices y el producto de un escalar por una matriz.

Finalmente escribe varias funciones en la pizarra que deberán ser resueltas por los estudiantes (correspondiendo a la tarea 3, que no discutimos aquí).

En este episodio, de aplicación de los contenidos previos, podemos ver como Jordy pretende que sus alumnos usen la multiplicación de matrices a un nivel puramente algorítmico, de manera que dicho proceso adquiere la naturaleza de artefacto de naturaleza matemática (HENRÍQUEZ; MONTOYA, 2015), para ser usado en un contexto de funciones matriciales, en el que el producto de matrices es una herramienta. Este uso induce una génesis instrumental, ya que se pretende hacer útil el artefacto en un contexto. En este sentido, Jordy parece estar usando, y por tanto conoce, una conexión de tipo auxiliar (KSM) (ESCUDEROÁVILA et al., 2015), que le da pie a conectar dos temas matemáticos (matrices y funciones), desde una perspectiva instrumental (uno de estos contenidos es usado como herramienta en el desarrollo de otro contenido). Podemos inferir que Jordy posee un KoT que le permite generar estos ejemplos, y que sabe calcular los productos involucrados. Este conocimiento tiene naturaleza tanto conceptual, en cuanto a comprender el algoritmo y sus propiedades, como procedimental, en cuanto a las habilidades operativas, y permea el tipo de actividad que Jordy fomenta en el aula.

Por otro lado, observamos cómo, al plantear la necesidad de multiplicar -3 por la matriz identidad, se pretende desarrollar la génesis discursiva, dotando de coherencia a los elementos del referencial, y que, a través de dicha génesis, los alumnos comprendan que -3, como sumando, en el contexto de una función de matrices de naturaleza polinómica, tiene el 
significado de -3 multiplicado por la identidad. Esto, a su vez, parece pretender reforzar la visualización de los alumnos, que usando su conocimiento del referencial han de dotar de significado los elementos del representamen en este contexto determinado (-3 pasa a ser interpretado como el producto de un escalar por la matriz identidad correspondiente). Todo este proceso se sustenta en el KoT del profesor, acerca de cuándo se puede realizar la suma de matrices y que no está definida la suma de una matriz con un escalar, y, por otra parte, parece mostrar un conocimiento de algunas propiedades de las funciones matriciales, que le permiten afirmar que como imagen de la función se debe obtener otra matriz.

\section{Conclusiones}

En este artículo nos planteábamos cómo el análisis del conocimiento que sustenta la práctica de un profesor de Álgebra lineal (usando el modelo MTSK) podía verse enriquecido con la consideración de la actividad matemática que propiciaba el profesor, analizada en el marco del ETM. Para ello, hemos analizado una sesión de clase sobre la multiplicación de matrices. En los resultados del análisis observamos que el conocimiento matemático de este profesor (en este caso KoT y KSM), parece sustentar la activación de la génesis discursiva, así como la génesis instrumental. De este modo, por ejemplo, su conocimiento de propiedades de la multiplicación (como que, en general, no se da la propiedad conmutativa) le permite propiciar la argumentación sobre dicha propiedad y su justificación. Por otra parte, su KFLM puede explicar que Jordy enfatice determinados aspectos del contenido, enunciando tareas específicas y estructurando la sesión como lo hace. Con dicho énfasis, se propicia la activación de ciertas génesis (por ejemplo, la importancia atribuida a los errores de los alumnos por generalizar aspectos de la suma de matrices, le lleva a introducir una tarea que pone el énfasis en la condición necesaria sobre las dimensiones de las matrices para poder realizar su producto, lo que asociamos a que se favorezca la justificación sobre las características del procedimiento, ligado a la activación de la génesis discursiva). Finalmente, el KMT de Jordy muestra cómo se propone el trabajo para potenciar las distintas génesis, siendo el conocimiento que permite al profesor pasar de su reflexión acerca de lo que debería aprenderse, a la planificación acerca de cómo organizar la sesión, reflejándose en las tareas que propone (en este caso, en los ejemplos que elige en dichas tareas y en su presentación del contenido). De este modo, el MTSK del profesor parece explicar en parte el trabajo matemático que propone en el aula, esto es, el ETM idóneo del profesor. Por otro lado, el ETM, nos acerca a comprender en qué pone el énfasis el profesor en la actividad en el aula, 
permitiendo describir la enseñanza que implementa, y el tipo de reflexión que intenta generar en sus alumnos acerca del contenido matemático. Esta descripción está mediada, en gran parte, por la naturaleza del propio contenido. Así, por ejemplo, en el caso de Jordy, al analizar clases de álgebra lineal, observamos un gran énfasis en lo procedimental.

Entendemos que el estudio del conocimiento especializado del profesor es útil a la hora de establecer los recursos con los que éste gestiona su espacio de trabajo matemático, es decir, ambos modelos pueden coordinarse y enriquecerse mutuamente cuando se trata del estudio de la actividad matemática en el aula. La imagen obtenida del ETM nos ha permitido determinar dónde pone el énfasis el profesor en la actividad matemática que promueve en el aula. El MTSK nos ha permitido aproximarnos a su conocimiento. Y el doble análisis, ETM y MTSK nos permite establecer relaciones entre la actividad matemática que el profesor propone y el conocimiento matemático que sustenta dicha actividad. En el caso de la práctica de Jordy, en la sesión analizada, apenas se percibe la intervención de los alumnos, por lo que resulta imposible indagar sobre el ETM de éstos, y su relación con el ETM idóneo. Creemos que esto permitiría vislumbrar otro conjunto de relaciones entre el ETM idóneo y el MTSK del profesor, que nos podría ayudar a comprender cómo interpreta el propio profesor el ETM de los estudiantes, y cómo modifica el ETM idóneo en función de dicha interpretación.

Para estudiar el papel del conocimiento del profesor en el diseño, gestión, y evaluación de los ETM, y en pos de una caracterización sistemática, creemos interesante la posibilidad de establecer un proceso de Networking (BIKNER-AHSBAHS et al., 2010), de manera que las relaciones emergentes entre ETM y MTSK, tanto en este artículo, como en Flores-Medrano et al. (en este mismo volumen) supongan una primera aproximación a este proceso. A priori, entendemos que las limitaciones de este proceso para coordinar ambos constructos teóricos emergerán de las limitaciones de ambos modelos. Por ejemplo, el modelo ETM va ligado a la naturaleza del contenido, lo que implicará una matización para cada contenido matemático, como en este caso la naturaleza procedimental del tratamiento del álgebra de matrices. Este tipo de trabajo de coordinación teórica permitiría, a través de la comprensión de cómo ambos marcos teóricos se combinan e interrelacionan, dar pie a la comprensión de la actividad de aula desde la perspectiva del ETM desarrollado por el alumno y del conocimiento usado por el profesor, y de cómo el conocimiento del profesor le permite gestionar la actividad del alumno. Este proceso tiene el potencial de permitir profundizar en el estudio de la interacción entre conocimiento del profesor y tipo de trabajo desarrollado por el alumno, completando la comprensión de la actividad de aula. 


\section{Agradecimientos}

Los autores son miembros del proyecto de investigación "Caracterización del Conocimiento Especializado del Profesor de Matemáticas” (EDU2013-44047P), financiado por el Ministerio de Economía y Competitividad. Gobierno de España.

\section{Referencias}

BIKNER-AHSBAHS, A. et al. Networking of theories in mathematics education (Research Forum). In: PROCEEDINGS OF THECONFERENCE OF THE INTERNATIONAL GROUP FOR THE PSYCHOLOGY OF MATHEMATICS EDUCATION, 34, 2010, Belo Horizonte. Proceedings of PME 34. Belo Horizonte: PME, 2010. v. 1, p.145-175.

BRYMAN. A. Social Research Methods. 2. ed. New York: Oxford University Press, 2011.

CARRILLO, J. et al. Determining specialised knowledge for mathematics teaching. In: PROCEEDINGS OF THE CONGRESS OF THE EUROPEAN SOCIETY FOR RESEARCH IN MATHEMATICS EDUCATION, 8, 2013, Antalya. Proceedings of CERME 8. Antalya: ERME, 2013. p. 2985-2994.

CHARALAMBOUS, C.Y. Mathematical Knowledge for Teaching and Providing Explanations: an Exploratory Study. In: PROCEEDINGS OF THE CONFERENCE OF THE INTERNATIONAL GROUP FOR THE PSYCHOLOGY OF MATHEMATICS EDUCATION, $34^{\text {rd }}, 2009$, Thessaloniki. Proceedings of PME 34. Thessaloniki: PME, 2009, v. 2. p. 305-312.

DUVAL, R. Sémiosis et pensée humaine. registres sémiotiques et apprentissages intellectuels. Berne: Peter Lang, 1995.

ESCUDERO-ÁVILA, D. I. et al.. El conocimiento especializado del profesor de matemáticas detectado en la resolución del problema de las cuerdas. PNA, Granada, v. 10, n. 1, p. 53-77, sep. 2015.

FIGUEIREDO, C.; CONTRERAS, L.C.; BLANCO, L. A transparencia e a variação dos exemplos utilizados na aprendizagem de conceitos matemáticos. Revista Zetetiké FE/UNICAMP, Campinas, v. 17, n. 32, p. 29-60, jul. 2009.

FLORES-MEDRANO, E. et al. El Papel del MTSK como modelo de conocimiento del profesor en las interrelaciones entre los espacios de trabajo matemático. Bolema, Rio Claro (SP). Trabajo en revisión.

HENRÍQUEZ, C.; MONTOYA, E. Espacios de trabajo geométrico sintético y analítico de profesores y su práctica en el aula. Enseñanza de las Ciencias, Barcelona, v. 33, n.2, p. 51-70, feb. 2015.

KUZNIAK, A. L'espace de travail mathématique et ses genèses. Annales de Didactique et de Sciences Cognitives, Strasbourg, v. 16, n. único, p. 9-24. 2011.

KUZNIAK, A.; RICHARD, P. Espacios de trabajo matemático. Puntos de vista y perspectivas.

Revista Latinoamericana de Investigación en Matemática Educativa, México, v. 17, n. 4-I, p. 5-15, dic. 2014.

KUZNIAK, A.; NECHACHE, A. Using the geometric working spaces in order to plan the teaching of geometry. In: PROCEEDINGS OF THE CONGRESS OF EUROPEAN SOCIETY FOR RESEARCH IN MATHEMATICS EDUCATION, 9, 2016, Prague. Proceedings of CERME 9. Prague: ERME, in press. 
LAVAL, D. L'algorithmique comme objet d'apprentissage de la démarche de preuve en théorie élémentaire des nombres: algorithme de Kaprekar. In: QUATRIÈME SYMPOSIUM ESPACE DE TRAVAIL MATHÉMATIQUE, 4, 2015, San Lorenzo de El Escorial. Actes du ETM 4. San Lorenzo de El Escorial: Universidad Complutense de Madrid, 2015, p. 103-115.

MARTÍNEZ, M. et al. El conocimiento del horizonte matemático: más allá de conectar el presente con el pasado y el futuro. In: SIMPOSIO DE LA SOCIEDAD ESPAÑOLA DE INVESTIGACIÓN EN EDUCACIÓN MATEMÁTICA, 15, 2011, Ciudad Real. Actas de SEIEM 15. Ciudad Real: Universidad de Castilla-La Mancha, 2011. p. 429-438.

MONTES, M. A. et al. MTSK: From common and horizon knowledge to knowledge of topics and structures. In: PROCEEDINGS OF THE CONGRESS OF THE EUROPEAN SOCIETY FOR RESEARCH IN MATHEMATICS EDUCATION, 8, 2013, Antalya. Proceedings of CERME 8. Antalya: ERME, 2013. p. 3185-3194.

ROJAS, N; FLORES, P.; CARRILLO, J. Conocimiento especializado de un profesor de matemáticas de educación primaria al enseñar los números racionales. Bolema, Rio Claro (SP), v. 29, n. 51, p. 143 167, abr. 2015.

SÁNCHEZ, M. A review of research trends in mathematics teacher education. PNA, Granada, v. 5, n.4, p. 129-145, jun. 2011.

VASCO, D. et al. The characterisation of the specialised knowledge of a university lecturer in linear algebra. En: PROCEEDINGS OF THE CONGRESS OF EUROPEAN SOCIETY FOR RESEARCH IN MATHEMATICS EDUCATION, 9, 2016, Prague. Proceedings of CERME 9. Prague: ERME, in press.

VASCO, D. et al.. Specialised knowledge of a linear algebra at the university level. En: PROCEEDINGS OF THE CONFERENCE OF THE INTERNATIONAL GROUP FOR THE PSYCHOLOGY OF MATHEMATICS EDUCATION, 38, 2014, Vancouver. Proceedings of PME 38. Vancouver: PME, 2014, v. 6, p. 401.

YIN, R.K. Case study research: design and methods. London: Sage, 1989.

Submetido em Julho de 2015. Aprovado em Setembro de 2015. 